\title{
Limit theorems for radial random walks on homogeneous spaces with growing dimensions
}

\author{
Michael Voit \\ Preprint 2008-18 \\ Oktober 2008
}

Fakultät für Mathematik

Technische Universität Dortmund

Vogelpothsweg 87

44227 Dortmund 



\title{
Limit theorems for radial random walks on homogeneous spaces with growing dimensions
}

\author{
Michael Voit \\ Fachbereich Mathematik, Technische Universität Dortmund \\ Vogelpothsweg 87, D-44221 Dortmund, Germany \\ E-mail: michael.voit@math.uni-dortmund.de
}

\begin{abstract}
Let $X_{p}=G_{p} / K_{p}$ be homogeneous spaces ( $K_{p}$ a compact subgroup of a locally compact group $G_{p}$ ) with dimension parameter $p$ such that the double coset spaces $G_{p} / / K_{p}$ can be identified with some fixed space $X$. Then we obtain projections $T_{p}: X_{p} \rightarrow X$, and for a given probability measure $\nu \in M^{1}(X)$ there exist unique "radial", i.e. $K_{p}$-invariant measures $\nu_{p} \in M^{1}\left(X_{p}\right)$ with $T_{p}\left(\nu_{p}\right)=\nu$ and associated radial random walks $\left(S_{n}^{p}\right)_{n}$ on the $X_{p}$. We generally ask for limit theorems for $T_{p}\left(S_{n}^{p}\right)$ for $n, p \rightarrow \infty$. In particular we give a survey about existing results for the Euclidean spaces $X_{p}=\mathbb{R}^{p}$ (with $K_{p}=S O(p)$ and $X=[0, \infty[))$ and matrix spaces, and we derive a new central limit theorem for the hyperbolic spaces $X_{p}$ of dimensions $p$ over $\mathbb{F}=\mathbb{R}, \mathbb{C}, \mathbb{H}$.
\end{abstract}

KEYWORDS: Radial random walks, hyperbolic spaces, Jacobi functions, central limit theorems, large dimensions, Bessel convolution, Jacobi convolution

\section{Introduction}

In the theory of classical limit theorems for random walks, one usually considers a (say timehomogeneous) Markov chain $\left(S_{n}\right)_{n \geq 0}$ on a fixed state space and is interested in limit theorems for functionals of $S_{n}$ for $n \rightarrow \infty$. On the other hand, e.g., in random matrix theory, often different limit theorems are considered. Here one has a sequence $\left(X_{p}\right)_{p \geq 1}$ of state spaces, a sequence of probability measures $\nu_{p} \in M^{1}\left(X_{p}\right)$, and a sequence of mappings $T_{p}: X_{p} \rightarrow X$ into a fixed space $X$ of characteristics. Here one may ask for limit theorems for the distributions $T_{p}\left(\nu_{p}\right) \in M^{1}(X)$ for $p \rightarrow \infty$. A famous typical example is given by the the largest eigenvalues of a Gaussian unitary ensemble where the $\nu_{p} \in M^{1}\left(X_{p}\right)$ are the distributions on the spaces $X_{p}$ of all $p \times p$ complex Hermitian matrices whose entries are independent centered Gaussians with suitable normalizations, where the Tracy-Widom distribution appears as limit, see e.g. [18] for a survey.

It is an interesting general task to combine both problems: Let $\left(X_{p}\right)_{p \geq 1}$ be a sequence of state spaces with a sequence of measurable mappings $T_{p}: X_{p} \rightarrow X$ into a fixed space $X$ of characteristics. For each dimension parameter $p$ consider a time-homogeneous Markov-chain $\left(S_{n}^{p}\right)_{n \geq 0}$ on $X_{p}$ such that the $S_{n}^{p}$ are coupled in a suitable way. We now ask for limit theorems for $T_{p}\left(S_{n}^{p}\right)$ for $n, p \rightarrow \infty$ in a suitably coupled way.

This is particularly interesting for series of homogeneous spaces $X_{p}=G_{p} / K_{p}$ with compact subgroups $K_{p}$ of locally compact groups $G_{p}$, where the double coset spaces $G_{p} / / K_{p}$ may be identified with a space $X$ independent of $p$. Then one has canonical mappings 
$T_{p}: X_{p}=G_{p} / K_{p} \rightarrow G_{p} / / K_{p} \simeq X$, and for a fixed probability measure $\nu \in M^{1}(X)$ one has a unique radial (i.e., $K_{p}$-invariant) measure $\nu_{p} \in M^{1}(X)$ with $T_{p}\left(\nu_{p}\right)=\nu$. In this way one can introduce time-homogeneous radial (i.e., $K_{p}$-invariant) random walks $\left(S_{n}^{p}\right)_{n \geq 0}$ associated with the $\nu_{p}$ by $S_{0}^{p}:=K_{p} \in X_{p}$ and

$$
P\left(S_{n+1}^{p} \in A K_{p} \mid S_{n}^{p}=x K_{p}\right)=\nu_{p}\left(x^{-1} A K_{p}\right)=\nu\left(K_{p} x^{-1} A K_{p}\right)
$$

for $n \geq 0, x \in G_{p}$, and $A \subset G_{p}$ a Borel set. In this setting, one now may ask for limit theorems for the $X$-valued random variables $T_{p}\left(S_{n}^{p}\right)$ for $n, p \rightarrow \infty$. It turns out that one obtains fundamentally different results for the compact and non-compact case.

In the compact case, the Kawada-Ito theorem yields under natural assumptions that for fixed $p$ and $n \rightarrow \infty$, the distributions of $S_{n}^{p}$ tend the uniform distribution $\omega_{p}$ on $X_{p}$, and it is an interesting task to study the total variation distances $d(p, n):=\left\|P_{S_{n}^{p}}-\omega_{p}\right\|$ for $n, p \rightarrow \infty$. It is well-known that in many cases so-called cutoff-phenomena appear (see [3] for a survey), i.e., one finds an increasing sequence $n(p)$ such that roughly for $p \rightarrow \infty$, $d(p, n) \rightarrow 0$ for $n>n(p)$ and $d(p, n) \rightarrow 1$ for $n<n(p)$. For particular examples, this cutoff phenomenon can be explained in a precise quantitative way via limit theorems for $T_{p}\left(S_{n(p)}^{p}\right)$ (for particular sequences $\left.(n(p))_{p}\right)$ and $T_{p}\left(\omega_{p}\right)$ and comparing the different limits. This was done, for instance, for random walks associated the Ehrenfest urn and Hamming schemes in [4], [9], [21], for random walks associated with the Johnson scheme in [10], and for the spheres $S^{p} \subset \mathbb{R}^{p+1}$ and projective spaces over $\mathbb{F}=\mathbb{R}, \mathbb{C}$ or the quaternions $\mathbb{H}$ in [20], [22], and [23]. We also mention that for finite graphs $X_{p}$ such limit theorems are related with the spectral theoretic point of view in [11].

In this paper we focus on the noncompact case where only a few examples are discussed up to now. We consider the following three examples:

(1) Let $X_{p}=\mathbb{R}^{p}, K_{p}=S O(p)$, and $G_{p}:=S O(p) \times \mathbb{R}^{p}$ the motion group acting on $\mathbb{R}^{p}$. Then $X_{p} \simeq G_{p} / K_{p}$ and $G_{p} / / K_{p} \simeq\left(\mathbb{R}^{p}\right)^{S O(p)} \simeq[0, \infty[$ in a canonical way. Let $\nu \in M^{1}(([0, \infty[)$ be a fixed probability measure. Then for each dimension $p \in \mathbb{N}$ there is a unique radial (i.e., $S O(p)$-invariant) probability measure $\nu_{p} \in M^{1}\left(\mathbb{R}^{p}\right)$ with $\nu$ as its radial part. Consider i.i.d. $\mathbb{R}^{p}$-valued random variables $X_{k}^{p}, k \in \mathbb{N}$, with law $\nu_{p}$ and the associated radial random walks $\left(S_{n}^{p}:=\sum_{k=1}^{n} X_{k}^{p}\right)_{n \geq 0}$ on $\mathbb{R}^{p}$. One now might ask for limit theorems for the $\left[0, \infty\left[\right.\right.$-valued random variables $\left\|S_{n}^{p}\right\|_{2}$ for $n, p \rightarrow \infty$. Some answers (laws of large numbers, central limit theorems) are given in [17], [19], [25] and will be reviewed in Section 2 .

(2) The examples in (1) admit a higher rank extension: Fix the field $\mathbb{F}=\mathbb{R}, \mathbb{C}$ or $\mathbb{H}$ and a dimension $q \geq 1$ (where case (1) appears for $q=1$ ). Consider the vector spaces $X_{p}=M_{p, q}(\mathbb{F})$ of $p \times q$-matrices over $\mathbb{F}$ together with the mappings $\varphi_{p}: X_{p} \rightarrow \Pi_{q}, \quad x \mapsto$ $\left(x^{*} x\right)^{1 / 2}$ into the cone $\Pi_{q}=\Pi_{q}(\mathbb{F})$ of positive semidefinite $q \times q$-matrices over $\mathbb{F}$. We fix a probability measure $\nu \in M^{1}\left(\Pi_{q}\right)$. It can be checked by uniqueness of polar decomposition that for each $p$ there is a unique $U_{p}$-invariant distribution $\nu_{p} \in M^{1}\left(X_{p}\right)$ with $\varphi_{p}\left(\nu_{p}\right)=\nu$. We now again consider i.i.d. $\mathbb{R}^{p}$-valued random variables $X_{k}^{p}, k \in \mathbb{N}$, with law $\nu_{p}$ as well as the associated radial random walks $\left(S_{n}^{p}:=\sum_{k=1}^{n} X_{k}^{p}\right)_{n \geq 0}$ on $\mathbb{R}^{p}$, and one might ask for limit theorems for the $\Pi_{q}$-valued random variables $\varphi_{p}\left(S_{n}^{p}\right)$ for $n, p \rightarrow \infty$. Similar answers as in (1) are given in [17], [25], and will be reviewed in Section 3 below.

(3) Let again $\mathbb{F}=\mathbb{R}, \mathbb{C}$, or $\mathbb{H}$. For $p \geq 2$ consider the hyperbolic space $X_{p}=G_{p} / K_{p}$ of 
dimension $p$ over $\mathbb{F}$ with

$$
\begin{array}{ll}
\mathbb{F}=\mathbb{R}: & G_{p}=S O_{o}(1, p), \quad K_{p}=S O(p) \\
\mathbb{F}=\mathbb{C}: & G_{p}=S U(1, p), \quad K_{p}=S(U(1) \times U(p)) \\
\mathbb{F}=\mathbb{H}: & G_{p}=S p(1, p), \quad K_{p}=S p(1) \times S p(p) .
\end{array}
$$

For these noncompact symmetric spaces of rank 1, the hyperbolic distances lead to canonical homeomorphisms between $\varphi: G_{p} / / K_{p}$ and $[0, \infty$ [. If we fix a probability measure $\nu \in M^{1}([0, \infty[)$ as well as $p$, we obtain a unique associated radial distribution $\nu_{p} \in M^{1}\left(X_{p}\right)$ as well as an associated radial random walk $\left(S_{n}^{p}\right)_{n \geq 0}$ on $X_{p}$ as described above. In Section 4 of this paper we shall derive a central limit theorem for $n \rightarrow \infty$ and $n^{2} / p \rightarrow 0$.

It is clear that there exist many further interesting examples.

We briefly turn to the proofs. All proofs (in the compact and noncompact case) are based on asymptotic results for the spherical functions on the spaces $X_{p}$ or asymptotic results on the double coset convolutions on $G_{p} / / K_{p}=X$ for $p \rightarrow \infty$ (or a combination of both methods). For instance, in case (1) above, the spherical functions are Bessel functions, and the double coset convolutions are the well-known Bessel convolutions (see e.g. [14]). Moreover, in case (2), Bessel functions of matrix argument and the associated Bessel convolutions on matrix cones appear (see [6], [8], [16]), and in case (3), Jacobi functions and Jacobi convolutions on $[0, \infty[$ appear (see the survey [15]). Finally, in the compact case, spheres and projective spaces lead to Jacobi polynomials and Jacobi convolutions on $[-1,1]$, and the finite examples associated with Hamming schemes or Johnson schemes lead to Krawtchouk and Meixner polynomials respectively.

All examples have in common that the associated spherical are special functions depending on a discrete series of dimension parameters, which admit a product formula associated with the double coset convolution, and that these product formulas and the associated convolution may be extended to a continuous family of parameters. In this way one obtains continuous families of commutative hypergroup structures $\left(X ; *_{p}\right)$ with these special functions are characters, which generalize discrete families of double coset hypergroups; see [2] and [13] for the theory and notion of hypergroups. The hypergroup convolution $*_{p}$ on $X$ allows to introduce the notion of random walks on $(X, *)$ by saying that a (time-homogeneous) Markov $\left(Z_{n}^{p}\right)_{n \geq 0}$ is a random walk on $\left(X ; *_{p}\right)$ with law $\nu \in M^{1}(X)$ if

$$
P\left(Z_{n+1}^{p} \in A \mid Z_{n}^{p}=x\right)=\left(\delta_{x} * \nu\right)(A)
$$

for all $n \geq 0, x \in X$, and Borel sets $A \subset X$. It can be checked easily that for a parameter $p$ belonging to the homogeneous space $X_{p}=G_{p} / K_{p}$, and for a radial random walk $\left(S_{n}^{p}\right)_{n \geq 0}$ on $X_{p}$ as discussed in Eq. (1.1), the projection $\left(T_{p}\left(S_{n}^{p}\right)\right)_{n \geq 0}$ becomes a random walk on $\left(X, *_{p}\right)$ as in $\left((1.2)\right.$. In this way, many limit theorems for the $T_{p}\left(S_{n}^{p}\right)$ can be embedded into limit theorems for the random variables $Z_{n}^{p}$ on the hypergroups for $n, p \rightarrow \infty$ without additional effort. We thus state most results in this context.

\section{$2 \quad$ Radial limit theorems on $\mathbb{R}^{p}$ for $p \rightarrow \infty$}

Let $\nu \in M^{1}([0, \infty[)$ be a fixed probability measure. Then for each dimension $p \in \mathbb{N}$ there is a unique radial probability measure $\nu_{p} \in M^{1}\left(\mathbb{R}^{p}\right)$ with $\nu$ as its radial part, i.e., $\nu$ is the 
image of $\nu_{p}$ under the norm mapping $\varphi_{p}(x):=\|x\|_{2}$. For each $p \in \mathbb{N}$ consider i.i.d. $\mathbb{R}^{p}$-valued random variables $X_{k}^{p}, k \in \mathbb{N}$, with law $\nu_{p}$ as well as the associated radial random walks

$$
\left(S_{n}^{p}:=\sum_{k=1}^{n} X_{k}^{p}\right)_{n \geq 0}
$$

on $\mathbb{R}^{p}$. According to the philosophy of the introduction, we are interested in limit theorems for the $\left[0, \infty\right.$ [-valued random variables $\left\|S_{n}^{p}\right\|_{2}$ for $n, p \rightarrow \infty$. In [19] we proved that for all sequences $p_{n} \rightarrow \infty$,

$$
\left\|S_{n}^{p_{n}}\right\|_{2}^{2} / n \rightarrow \sigma^{2}:=\sigma^{2}(\nu):=\int_{0}^{\infty} x^{2} d \nu(x)
$$

in law under the condition $\sigma^{2}<\infty$. Moreover, in [17] an associated strong law and a large deviation principle were derived under the condition that $p_{n}$ grows fast enough. As the precise conditions are a little bit complicated, we here omit details and turn to central limit theorems. It turns out that two different results appear for $p>>n$ and $n>>p$. For $n>>p$, the multidimensional Berry-Esseen inequality on $\mathbb{R}^{p}$ of Bentkus [1] with an explicit constant depending on $p$ leads to the following result (see [25]):

2.1 Theorem. Assume that $\nu \in M^{1}\left(\left[0, \infty[)\right.\right.$ with $\nu \neq \delta_{0}$ admits a finite third moment $m_{3}(\nu):=\int_{0}^{\infty} x^{3} d \nu(x)<\infty$, and that $\lim _{n \rightarrow \infty} n / p_{n}^{3}=\infty$. Then

$$
\frac{\sqrt{p}_{n}}{n \sigma^{2} \sqrt{2}}\left(\left\|S_{n}^{p_{n}}\right\|_{2}^{2}-n \sigma^{2}\right)
$$

tends in distribution for $n \rightarrow \infty$ to the standard normal distribution $N(0,1)$.

For $p>>n$, the following result was established in [25]:

2.2 Theorem. Assume that $\nu \in M^{1}\left(\left[0, \infty[)\right.\right.$ admits a finite fourth moment $m_{4}(\nu):=$ $\int_{0}^{\infty} x^{4} d \nu(x)<\infty$, and that $\lim _{n \rightarrow \infty} n^{2} / p_{n} \rightarrow 0$. Then

$$
\frac{\left\|S_{n}^{p_{n}}\right\|_{2}^{2}-n \sigma^{2}}{\sqrt{n}}
$$

tends in distribution for $n \rightarrow \infty$ to $N\left(0, m_{4}(\nu)-\sigma^{4}\right)$.

The proof of this theorem in [25] is based on the observation that for fixed $p,\left(\left\|S_{n}^{p}\right\|_{2}\right)_{n \geq 0}$ is a random walk on $\left[0, \infty\left[\right.\right.$ associated with the Bessel-Kingman hypergroup structure $\left(\left[0, \infty\left[, *_{\alpha}\right)\right.\right.$ of index $\alpha:=p / 2-1$, where the convolution is generated by

$$
\delta_{x} *_{\alpha} \delta_{y}(f):=\frac{\Gamma(\alpha+1)}{\Gamma(\alpha+1 / 2) \sqrt{\pi}} \int_{-1}^{1} f\left(\sqrt{x^{2}+y^{2}+2 x y t}\right) \cdot\left(1-t^{2}\right)^{\alpha-1 / 2} d t
$$

for $f \in C_{b}([0, \infty[), \alpha>-1 / 2, x, y \geq 0$, and that for $\alpha \rightarrow \infty$,

$$
\delta_{x} *_{\alpha} \delta_{y}(f) \rightarrow f\left(\sqrt{x^{2}+y^{2}}\right) .
$$

this limit is the key for the proof of Theorem 2.2 and works for all random walks on the Bessel-Kingman hypergroups $\left(\left[0, \infty\left[, *_{\alpha}\right)\right.\right.$ and not just for the group parameters $\alpha:=p / 2-1$. 


\section{Radial limit theorems on the matrix spaces $M_{p, q}$ for $p \rightarrow \infty$}

For fixed dimensions $p, q \in \mathbb{N}$ let $M_{p, q}=M_{p, q}(\mathbb{F})$ be the space of $p \times q$-matrices over $\mathbb{F}=\mathbb{R}, \mathbb{C}$ or the quaternions $\mathbb{H}$ with real dimension $d=1,2$ or 4 respectively. This is a Euclidean vector space of real dimension $d p q$ with scalar product $\langle x, y\rangle=\mathfrak{R} \operatorname{tr}\left(x^{*} y\right)$ where $x^{*}:=\bar{x}^{t}$, $\Re t:=\frac{1}{2}(t+\bar{t})$ is the real part of $t \in \mathbb{F}$, and $t r$ is the trace in $M_{q}:=M_{q, q}$. A measure on $M_{p, q}$ is called radial if it is invariant under the action of the unitary group $U_{p}=U_{p}(\mathbb{F})$ by left multiplication, $U_{p} \times M_{p, q} \rightarrow M_{p, q},(u, x) \mapsto u x$. This action is orthogonal w.r.t. the scalar product above, and, by uniqueness of the polar decomposition, two matrices $x, y \in M_{p, q}$ belong to the same $U_{p}$-orbit if and only if $x^{*} x=y^{*} y$. Thus the space $M_{p, q}^{U_{p}}$ of $U_{p}$-orbits in $M_{p, q}$ is naturally parameterized by the cone $\Pi_{q}=\Pi_{q}(\mathbb{F})$ of positive semidefinite $q \times q$ matrices over $\mathbb{F}$. We identify $M_{p, q}^{U_{p}}$ with $\Pi_{q}$ via $U_{q} x \simeq\left(x^{*} x\right)^{1 / 2}$, i.e., the canonical projection $M_{p, q} \rightarrow M_{p, q}^{U_{p}}$ will be realized as the mapping

$$
\varphi_{p}: M_{p, q} \rightarrow \Pi_{q}, \quad x \mapsto\left(x^{*} x\right)^{1 / 2} .
$$

The square root is used here in order to ensure for $q=1$ and $\mathbb{F}=\mathbb{R}$ that the setting above with $\Pi_{1}=\left[0, \infty\left[\right.\right.$ and $\varphi_{p}(x)=\|x\|$ appears. By taking images of measures, $\varphi_{p}$ induces a Banach space isomorphism between the space $M_{b}^{U_{q}}\left(M_{p, q}\right)$ of all bounded radial Borel measures on $M_{p, q}$ and the space $M_{b}\left(\Pi_{q}\right)$ of bounded Borel measures on the cone $\Pi_{q}$. In particular, for each $\nu \in M^{1}\left(\Pi_{q}\right)$ there is a unique radial probability measure $\nu_{p} \in M^{1}\left(M_{p, q}\right)$ with $\varphi_{p}\left(\nu_{p}\right)=\nu$.

As in the case $q=1$, we now consider for each $p \in \mathbb{N}$ i.i.d. $M_{p, q}$-valued random variables $X_{k}^{p}, k \in \mathbb{N}$, with law $\nu_{p}$ and the associated radial random walks $\left(S_{n}^{p}:=\sum_{k=1}^{n} X_{k}^{p}\right)_{n \geq 0}$.

3.1 Definition. We say that $\nu \in M^{1}\left(\Pi_{q}\right)$ admits a $k$-th moment $(k \in \mathbb{N})$ if

$$
m_{k}(\nu):=\int_{\Pi_{q}}\|s\|^{k} d \nu(s)<\infty
$$

where $\|s\|=\left(t r s^{2}\right)^{1 / 2}$ is the Hilbert-Schmidt norm. If the second moment exists, the second moment of $\nu$ is defined as the matrix-valued integral

$$
\sigma^{2}:=\sigma^{2}(\nu):=\int_{\Pi_{q}} s^{2} d \nu(s) \in \Pi_{q} .
$$

With these notions, the following generalizations of Theorems 2.1 and 2.2 hold; see [25]:

3.2 Theorem. Assume that $m_{4}(\nu)<\infty$. Moreover, let $\lim _{n \rightarrow \infty} n / p_{n}^{4}=\infty$. Then

$$
\frac{\sqrt{p_{n}}}{n}\left(\varphi_{p_{n}}\left(S_{n}^{p_{n}}\right)^{2}-n \sigma^{2}\right)
$$

tends in distribution to some normal distribution $N\left(0, T^{2}\right)$ on the vector space $H_{q}$ of hermitian $q \times q$-matrices over $\mathbb{F}$ (with a covariance matrix $T^{2}=T^{2}\left(\sigma^{2}\right)$ described in the proof below for $\mathbb{F}=\mathbb{R})$.

3.3 Theorem. Assume that $m_{4}(\nu)<\infty$ and $\lim _{n \rightarrow \infty} n^{2} / p_{n}=0$. Then

$$
\frac{1}{\sqrt{n}}\left(\varphi_{p_{n}}\left(S_{n}^{p_{n}}\right)^{2}-n \sigma^{2}\right)
$$

tends in distribution to the normal distribution $N\left(0, \Sigma^{2}\right)$ on $H_{q}$ where $\Sigma^{2}$ is the covariance matrix of $\varphi_{p_{n}}\left(X_{1}^{p_{n}}\right)$ (which is independent of $\left.p_{n}\right)$. 
Again, the proof of Theorem 3.2 is based on a Berry-Esseen inequality on the Euklidean space $M_{p, q} \simeq \mathbb{R}^{d p q}$ of [1], while the proof of Theorem 3.3 relies on an explicit formula for the Bessel convolution on $\Pi_{q}$ due to Rösler [16]. With this convolution, the result follows Similar as for the case $q=1$ described in Section 2 .

\section{A central limit theorem for hyperbolic spaces and Jacobi convolutions on $[0, \infty[$}

Let $k \geq 2$, and $\mathbb{F}=\mathbb{R}, \mathbb{C}$, or $\mathbb{H}$. The hyperbolic space of dimension $k$ over $\mathbb{F}$ may be regarded as the symmetric space $G / K$ with

$$
\begin{array}{ll}
\mathbb{F}=\mathbb{R}: & G=S O_{o}(1, k), \quad K=S O(k) \\
\mathbb{F}=\mathbb{C}: & G=S U(1, k), \quad K=S(U(1) \times U(k)) \\
\mathbb{F}=\mathbb{H}: & G=S p(1, k), \quad K=S p(1) \times S p(k) .
\end{array}
$$

In all cases, the double coset $G / / K$ can be regarded as the interval $[0, \infty$ [ by identifying $t \geq 0$ with the double coset

$$
K a_{t} K \quad \text { with } \quad a_{t}=\left(\begin{array}{ccccc}
\operatorname{ch} t & 0 & \ldots & 0 & \operatorname{sh} t \\
0 & & & & 0 \\
\vdots & & I_{k-1} & & \vdots \\
0 & & & & 0 \\
\operatorname{sh} t & 0 & \ldots & 0 & \operatorname{ch} t
\end{array}\right) ;
$$

see [5]. Using this homeomorphism $\varphi: G / / K \rightarrow[0, \infty[$, the hyperbolic distance on $G / K$ is given by $d(x K, y K)=\varphi\left(K y^{-1} x K\right)$. We derive the following central limit theorem for $G$-invariant time-homogeneous random walks $\left(S_{n}^{k}\right)_{n \geq 0}$ on $G / / K$.

4.1 Theorem. Let $\left(k_{n}\right)_{n \geq 1} \subset \mathbb{N}$ be an increasing sequence of dimensions with $\lim _{n \rightarrow \infty} n / \sqrt{k}_{n}=$ 0 , and let $\nu \in M^{1}\left(\left[0, \infty[)\right.\right.$ with a finite second moment $\int_{0}^{\infty} x^{2} d \nu(x)<\infty$. For each dimension $k \geq 2$ consider a $G$-invariant time-homogeneous random walk $\left(S_{n}^{k}\right)_{n \geq 0}$ on $G / K$ such that for all $n, k$, the random variables $d\left(S_{n}^{k+1}, S_{n}^{k}\right)$ have distribution $\nu$. Then, $\rho:=\int_{0}^{\infty} \ln (\operatorname{ch} x) d \nu(x)<$ $\infty$ and $\sigma^{2}:=\int_{0}^{\infty}(\ln (\operatorname{ch} x))^{2} d \nu(x)<\infty$ exist, and

$$
\frac{d\left(S_{n}^{k_{n}}, S_{0}^{k_{n}}\right)-n \rho}{\sqrt{n}}
$$

tends in distribution for $n \rightarrow \infty$ to the normal distribution $N\left(0, \sigma^{2}-\rho^{2}\right)$.

We shall derive this CLT by considering the distributions of the random walks $\left(d\left(S_{n}^{k}, S_{0}^{k}\right)\right)_{n \geq 0}$ on the spaces $G / / K \simeq[0, \infty$ [ equipped with the associated double coset convolutions. These convolutions may be regarded as special cases of Jacobi convolutions on $[0, \infty[$ depending on indices $\alpha \geq \beta \geq-1 / 2$ which were investigated mainly by Flensted-Jensen and Koornwinder. In the following we refer to the survey [15] on this subject. For $\alpha>\beta>-1 / 2$, this convolution is given by

$$
\begin{aligned}
& \delta_{r} *(\alpha, \beta) \delta_{s}(f):=c_{(\alpha, \beta)} \int_{0}^{1} \int_{0}^{\pi} f\left(\operatorname{arch}\left|\operatorname{ch} r \cdot \operatorname{ch} s+t e^{i \varphi} \operatorname{sh} r \cdot \operatorname{sh} s\right|\right) \\
& \cdot\left(1-t^{2}\right)^{\alpha-\beta-1}(t \sin \varphi)^{2 \beta} t d t d \varphi
\end{aligned}
$$


with

$$
c_{(\alpha, \beta)}=\frac{2 \Gamma(\alpha+1)}{\Gamma(1 / 2) \Gamma(\alpha-\beta) \Gamma(\beta+1 / 2)}=\left(\int_{0}^{1} \int_{0}^{\pi}\left(1-t^{2}\right)^{\alpha-\beta-1}(t \sin \varphi)^{2 \beta} t d t d \varphi\right)^{-1}
$$

For $\alpha>\beta=-1 / 2$, the degenerate formula

$$
\delta_{r} *_{(\alpha,-1 / 2)} \delta_{s}(f):=c_{(\alpha,-1 / 2)} \int_{-1}^{1} f(\operatorname{arch}|\operatorname{ch} r \cdot \operatorname{ch} s+t \cdot \operatorname{sh} r \cdot \operatorname{sh} s|) \cdot\left(1-t^{2}\right)^{\alpha-1 / 2} d t
$$

appears with

$$
c_{(\alpha,-1 / 2)}=\frac{\Gamma(\alpha+1)}{\Gamma(1 / 2) \Gamma(\alpha+1 / 2)} .
$$

The case $\alpha=\beta$ can be transformed into the case $\beta=-1 / 2$ by some quadratic transformation (see Section 5.3 of [15]) and will not be considered here separately.

With this notion, the hyperbolic spaces $G / K$ and their associated double coset convolutions are related with the indices $\alpha, \beta$ by

$$
\alpha=d k / 2-1, \quad \beta=d / 2-1,
$$

where $d=1,2,4$ is the dimension of $\mathbb{F}$ over $\mathbb{R}$, and $k$ the dimension of $G / K$ as before.

Now fix $\alpha \geq \beta \geq-1 / 2$. It is well-known that the Jacobi convolution of point measures above can be extended uniquely in a weakly continuous, bilinear way to a probabilitypreserving convolution $*_{(\alpha, \beta)}$ on $M_{b}([0, \infty[)$, and that one obtains the so-called Jacobi-type hypergroup on $[0, \infty[$ in this way.

Now fix a probability measure $\nu \in M^{1}([0, \infty[)$, and consider a time-homogeneous random walk $\left(S_{n}^{(\alpha, \beta)}\right)_{n \geq 0}$ on $[0, \infty[$ with law $\nu$ of index $(\alpha, \beta)$, i.e., a time-homogeneous Markov process starting at the identity 0 with transition probability

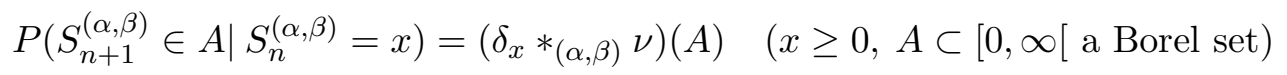

as described in the introduction. Theorem 4.1 above then can be regarded as a special case of the following central limit theorem:

4.2 Theorem. Let $\beta \geq-1 / 2$, and let $\left(\alpha_{n}\right)_{n \in \mathbb{N}} \subset[\beta, \infty[$ be an increasing sequence of indices with $\lim _{n \rightarrow \infty} n / \sqrt{\alpha}_{n}=0$. Let $\nu \in M^{1}\left(\left[0, \infty[)\right.\right.$ with a finite second moment $\int_{0}^{\infty} x^{2} d \nu(x)<\infty$. Then, $\rho:=\int_{0}^{\infty} \ln (\operatorname{ch} x) d \nu(x)<\infty$ and $\sigma^{2}:=\int_{0}^{\infty}(\ln (\operatorname{ch} x))^{2} d \nu(x)<\infty$ exist, and for the random walks $\left(S_{n}^{\left(\alpha_{n}, \beta\right)}\right)_{n \geq 0}$ on $[0, \infty[$,

$$
\frac{S_{n}^{\left(\alpha_{n}, \beta\right)}-n \rho}{\sqrt{n}}
$$

tends in distribution for $n \rightarrow \infty$ to $N\left(0, \sigma^{2}-\rho^{2}\right)$.

The proof is based on the observation that for $\alpha \rightarrow \infty$ and $f \in C([0, \infty[)$,

$$
\delta_{r} *_{(\alpha, \beta)} \delta_{s}(f) \quad \rightarrow \quad f(\operatorname{arch}(\operatorname{ch} r \cdot \operatorname{ch} s)) .
$$

The right hand side motivates to study a limit semigroup structure on $[0, \infty[$. We next introduce this limit structure and make the convergence above more precise. This then finally will lead to the proof of Theorem 4.2. 
4.3 Definition. (1) We equip the set $[0, \infty[$ with the commutative semigroup operation

$$
s \bullet r:=\operatorname{arch}(\operatorname{ch} r \cdot \operatorname{ch} s) \quad(r, s \geq 0),
$$

and extend this operation in a linear, weakly continuous way to the space of all bounded Borel measures by

$$
\nu_{1} \bullet \nu_{2}(f):=\int_{0}^{\infty} \int_{0}^{\infty} f(r \bullet s) d \nu_{1}(r) d \nu_{2}(s) .
$$

We here notice that the mapping $Q(x):=\ln \operatorname{ch} x$, establishes a semigroup isomorphism from $([0, \infty[, \bullet)$ onto the semigroup $([0, \infty[,+)$.

(2) A function $f \in C([0, \infty[)$ is called arche-Lipschitz continuous with constant $L$, if for all $x, y \geq 0$,

$$
\left|f\left(\operatorname{arch}\left(e^{x}\right)\right)-f\left(\operatorname{arch}\left(e^{y}\right)\right)\right| \leq L|x-y| .
$$

This notion is motivated by the fact, that for a function $f \in C_{0}([0, \infty[)$, which is Lipschitz continuous in the usual sense with constant $L$, the function $\tilde{f}(x):=f((Q(x))$ is arche-Lipschitz with the same $L$.

4.4 Lemma. Let $f \in C([0, \infty[)$ be arche-Lipschitz continuous with constant L. Then, for all $r, s \geq 0, t \in[0,1]$ and $\varphi \in[0, \pi]$,

$$
\begin{aligned}
& \left|f\left(\operatorname{arch}\left|\operatorname{ch} r \cdot \operatorname{ch} s+t e^{i \varphi} \operatorname{sh} r \cdot \operatorname{sh} s\right|\right)-f(\operatorname{arch}|\operatorname{ch} r \cdot \operatorname{ch} s|)\right| \\
& \leq 2 L \frac{t}{1-t^{2}} .
\end{aligned}
$$

Proof. By Definition 4.3(2), the left hand side of Eq. (4.3) is bounded above by

$$
\begin{aligned}
R & :=L \cdot\left|\ln \left(\left|\operatorname{ch} r \cdot \operatorname{ch} s+t e^{i \varphi} \operatorname{sh} r \cdot \operatorname{sh} s\right|\right)-\ln (\operatorname{ch} r \cdot \operatorname{ch} s)\right| \\
& =L \cdot|\ln (|1+x|)|
\end{aligned}
$$

with

$$
x:=t e^{i \varphi} \frac{\operatorname{sh} r \cdot \operatorname{sh} s}{\operatorname{ch} r \cdot \operatorname{ch} s}
$$

which satisfies $|x| \leq t$. Moreover, for $|1+x| \geq 1$, we have

$$
|\ln | 1+x|| \leq|x| \leq t .
$$

On the other hand, for $|1+x| \leq 1$, we first observe that by the mean value theorem,

$$
|\ln | 1+x|| \leq|x| /|1+x| .
$$

Therefore, using $\operatorname{ch} r \cdot \operatorname{ch} s-\operatorname{sh} r \cdot \operatorname{sh} s=\operatorname{ch}(r-s)$, we obtain

$$
\left|\operatorname{ch} r \cdot \operatorname{ch} s+t e^{i \varphi} \operatorname{sh} r \cdot \operatorname{sh} s\right| \geq \operatorname{ch} r \cdot \operatorname{ch} s-t \cdot \operatorname{sh} r \cdot \operatorname{sh} s=1+(1-t) \operatorname{sh} r \cdot \operatorname{sh} s,
$$

and thus

$$
|\ln | 1+x|| \leq t \cdot \frac{\operatorname{sh} r \cdot \operatorname{sh} s}{1+(1-t) \operatorname{sh} r \cdot \operatorname{sh} s} \leq t /(1-t) \leq 2 t /\left(1-t^{2}\right) .
$$

Combining (4.4), (4.5), and (4.6), we obtain the estimate in the lemma. 
4.5 Lemma. There is a constant $C=C(\beta)$ such that for all $r, s \in[0, \infty[$, all sufficiently large $\alpha$, and all arche-Lipschitz continuous functions $f$ on $[0, \infty[$ with constant $L$,

$$
\left|\delta_{r} *_{(\alpha, \beta)} \delta_{s}(f)-\delta_{r} \bullet \delta_{s}(f)\right| \leq C L / \sqrt{\alpha} .
$$

Proof. Assume that $\beta>-1 / 2$ holds. We use the definition of the Jacobi convolution above together with Lemma 4.4 and obtain

$$
\begin{aligned}
& \left|\delta_{r} *(\alpha, \beta) \delta_{s}(f)-\delta_{r} \bullet \delta_{s}(f)\right| \\
& \quad \leq c_{(\alpha, \beta)} \int_{0}^{1} \int_{0}^{\pi}\left|f\left(\operatorname{arch}\left|\operatorname{ch} r \cdot \operatorname{ch} s+t e^{i \varphi} \operatorname{sh} r \cdot \operatorname{sh} s\right|\right)-f(\operatorname{arch}|\operatorname{ch} r \cdot \operatorname{ch} s|)\right| \\
& \quad \leq 2 L c_{(\alpha, \beta)} \int_{0}^{1} \int_{0}^{\pi}\left(1-t^{2}\right)^{\alpha-\beta-2} t^{2 \beta+2} \sin ^{2 \beta} \varphi d t d \varphi \\
& \quad \leq C_{1} \frac{\Gamma(\alpha+1)}{\Gamma(\alpha-\beta)} \int_{0}^{1}\left(1-t^{2}\right)^{\alpha-\beta-2} t^{2 \beta+2} d t
\end{aligned}
$$

for some constant $C_{1}$. If we now use the beta integral

$$
\int_{0}^{1}\left(1-t^{2}\right)^{\alpha-\beta-2} t^{2 \beta+2} d t=\frac{\Gamma(\alpha-\beta-1) \Gamma(\beta+3 / 2)}{2 \Gamma(\alpha+1 / 2)} .
$$

as well as $\Gamma(\alpha+1) / \Gamma(\alpha+3 / 2)=O\left(\alpha^{-1 / 2}\right)$ for $\alpha \rightarrow \infty$, the statement of the lemma follows for $\beta>-1 / 2$. The proof for $\beta=-1 / 2$ is similar.

4.6 Lemma. There is a constant $C=C(\beta)$ such that for all arche-Lipschitz continuous functions $f$ on $\left[0, \infty\left[\right.\right.$ with constant $L$, all $\nu_{1}, \nu_{2} \in M^{1}([0, \infty[)$, and all sufficiently large $\alpha$,

$$
\left|\nu_{1} *_{(\alpha, \beta)} \nu_{2}(f)-\nu_{1} \bullet \nu_{2}(f)\right| \leq C L / \sqrt{\alpha}
$$

Proof. By Lemma 4.5,

$$
\begin{aligned}
\mid \nu_{1} *(\alpha, \beta) & \nu_{2}(f)-\nu_{1} \bullet \nu_{2}(f) \mid \\
& \leq \int_{0}^{\infty} \int_{0}^{\infty} \mid \delta_{r} *(\alpha, \beta) \\
& \leq \frac{C L}{\sqrt{\alpha}}
\end{aligned}
$$

4.7 Lemma. There is a constant $C=C(\beta)$ such that for all arche-Lipschitz continuous functions $f$ on $\left[0, \infty\left[\right.\right.$ with constant $L$, all $\nu_{1}, \nu_{2}, \nu_{3} \in M^{1}\left(\Pi_{q}\right)$ and all sufficiently large $\alpha$,

$$
\left|\left(\nu_{1} *_{(\alpha, \beta)} \nu_{2}\right) \bullet \nu_{3}(f)-\left(\nu_{1} \bullet \nu_{2}\right) \bullet \nu_{3}(f)\right| \leq C L / \sqrt{\alpha} .
$$

Proof. For $y \geq 0$, consider the function $f_{y}(x):=f(x \bullet y)$ on $[0, \infty[$. By the definition of $\bullet$ and our definition of arche-Lipschitz continuity, these translated functions $f_{y}$ are also arche-Lipschitz continuous with the same constant $L$. Therefore, by Lemma 4.6,

$$
\begin{aligned}
& \left|\left(\nu_{1} *(\alpha, \beta) \nu_{2}\right) \bullet \nu_{3}(f)-\left(\nu_{1} \bullet \nu_{2}\right) \bullet \nu_{3}(f)\right| \\
& \quad \leq \int_{0}^{\infty}\left|\int_{0}^{\infty} f_{y}(x) d\left(\nu_{1} *(\alpha, \beta) \nu_{2}\right)(x)-\int_{0}^{\infty} f_{y}(x) d\left(\nu_{1} \bullet \nu_{2}\right)(x)\right| d \nu_{3}(y) \\
& \quad \leq C L / \sqrt{\alpha}
\end{aligned}
$$


For $\nu \in M^{1}([0, \infty[)$ and $n \in \mathbb{N}$, we denote the $n$-fold convolution powers of $\nu$ w.r.t. the convolutions $*_{(\alpha, \beta)}$ and $\bullet$ by $\left.\nu^{(n, *(\alpha, \beta)}\right)$ and $\nu^{(n, \bullet)}$ respectively.

4.8 Proposition. Let $\nu \in M^{1}([0, \infty[)$. Then there is a constant $C=C(\beta)$ such that for all arche-Lipschitz continuous functions $f$ on $[0, \infty[$ with constant $L$, all $n \in \mathbb{N}$, and all sufficiently large $\alpha$,

$$
\left|\nu^{(n, *(\alpha, \beta)}(f)-\nu^{(n, \bullet)}(f)\right| \leq C L \frac{n}{\sqrt{\alpha}} .
$$

Proof. We observe that

$$
\begin{aligned}
\left.\mid \nu^{(n, *(\alpha, \beta)}\right)(f) & -\nu^{(n, \bullet)}(f) \mid \\
\leq & \left|\nu^{(n, *(\alpha, \beta)}(f)-\nu^{\left(n-1, *_{(\alpha, \beta)}\right)} \bullet \nu(f)\right| \\
& +\left|\left(\nu^{\left(n-2, *{ }_{(\alpha, \beta)}\right)} *(\alpha, \beta) \nu\right) \bullet \nu(f)-\nu^{(n-2, *(\alpha, \beta)} \bullet \bullet \bullet \nu(f)\right| \\
& +\ldots+\left|\left(\nu *_{(\alpha, \beta)} \nu\right) \bullet \nu^{(n-2, \bullet)}(f)-\nu^{(n, \bullet)}(f)\right|
\end{aligned}
$$

where by Lemma 4.7 , each summand of the right hand side is bounded by $C L / \sqrt{\alpha}$. This implies the proposition.

Proof of Theorem 4.2. Consider the isomorphism $Q(x):=\ln \operatorname{ch} x$ from $([0, \infty[, \bullet)$ onto $([0, \infty[,+)$. Let $f \in C_{0}(\mathbb{R})$ be Lipschitz-continuous in the usual sense on $\mathbb{R}$ with constant $L$. Then, for $n \in \mathbb{N}$, the function

$$
f_{n}(x):=f\left(\frac{Q(x)-n \rho}{\sqrt{n}}\right)
$$

on $[0, \infty]$ satisfies

$$
\left|f_{n}\left(\operatorname{arch} e^{x}\right)-f_{n}\left(\operatorname{arch} e^{y}\right)\right|=\left|f\left(\frac{x-n \rho}{\sqrt{n}}\right)-f\left(\frac{y-n \rho}{\sqrt{n}}\right)\right| \leq \frac{L|x-y|}{\sqrt{n}}
$$

i.e., $f_{n}$ is arche-Lipschitz with constant $n^{-1 / 2} L$. Therefore, by Proposition 4.8 and the assumptions of the theorem,

$$
\left.\mid \int_{0}^{\infty} f_{n} d \nu^{\left(n, *\left(\alpha_{n}, \beta\right)\right.}\right)-\int_{0}^{\infty} f_{n} d \nu^{(n, \bullet)} \mid \leq C L \frac{n}{\sqrt{\alpha}_{n}} \rightarrow 0
$$

for $n \rightarrow \infty$ with

$$
\int_{0}^{\infty} f_{n} d \nu^{\left(n, *_{\left(\alpha_{n}, \beta\right)}\right)}=\int_{0}^{\infty} f_{n} d P_{S_{n}^{\left(\alpha_{n}, \beta\right)}}=\int_{\mathbb{R}} f d P_{\left(Q\left(S_{n}^{\left(\alpha_{n}, \beta\right)}\right)-n \rho\right) / \sqrt{n}}
$$

where $P_{X}$ denotes the law of a random variable $X$. Moreover, as $Q$ is a isomorphism from $([0, \infty[, \bullet)$ onto $([0, \infty[,+)$, and denoting the classical convolution of measures on $([0, \infty[,+)$ by $*$, we see that

$$
\begin{aligned}
\int_{0}^{\infty} f_{n} d \nu^{(n, \bullet)} & =\int_{0}^{\infty} f\left(\frac{Q(x)-n \rho}{\sqrt{n}}\right) d \nu^{(n, \bullet)}(x) \\
& =\int_{0}^{\infty} f\left(\frac{x-n \rho}{\sqrt{n}}\right) d Q\left(\nu^{(n, \bullet)}\right)(x) \\
& =\int_{0}^{\infty} f\left(\frac{x-n \rho}{\sqrt{n}}\right) d\left(Q(\nu)^{(n, *)}\right)(x)
\end{aligned}
$$


where the latter tends by the classical central limit theorem on $\mathbb{R}$ to $\int_{\mathbb{R}} f d N\left(0, \sigma^{2}-\rho^{2}\right)$. Taking (4.7) and (4.8) into account, we obtain that

$$
\int_{\mathbb{R}} f d P_{\left(Q\left(S_{n}^{\left(\alpha_{n}, \beta\right)}\right)-n \rho\right) / \sqrt{n}} \rightarrow \int_{H_{q}} f d N\left(0, \sigma^{2}-\rho^{2}\right)
$$

for $n \rightarrow \infty$ and all Lipschitz-continuous functions $f \in C_{0}(\mathbb{R})$. As the space of Lipschitz continuous functions in $C_{0}(\mathbb{R})$ is $\|\cdot\|_{\infty}$-dense in $C_{0}(\mathbb{R})$, a simple $\varepsilon$-argument together with the triangle inequality ensures that Eq. (4.10) holds in fact for all $f \in C_{0}(\mathbb{R})$. Therefore,

$$
\frac{\ln \left(\operatorname{ch}\left(S_{n}^{\left(\alpha_{n}, \beta\right)}\right)\right)-n \rho}{\sqrt{n}}
$$

tends in distribution to $\left(N\left(0, \sigma^{2}-\rho^{2}\right)\right.$. This in particular shows that $\ln \left(\operatorname{ch}\left(S_{n}^{\left(\alpha_{n}, \beta\right)}\right)\right) / n \rightarrow \rho^{2}$ and thus $e^{-2 S_{n}^{\left(\alpha_{n}, \beta\right)}} \rightarrow 0$ in probability. Using

$$
x-\ln 2 \leq \ln (\operatorname{ch} x) \leq x+\ln \left(1+e^{-2 x}\right) \leq x+e^{-2 x}
$$

and thus

$$
\ln \operatorname{ch} S_{n}^{\left(\alpha_{n}, \beta\right)}-e^{-2 S_{n}^{\left(\alpha_{n}, \beta\right)}} \leq S_{n}^{\left(\alpha_{n}, \beta\right)} \leq \ln \operatorname{ch} S_{n}^{\left(\alpha_{n}, \beta\right)}+\ln 2,
$$

we obtain the theorem from Eq. (4.11).

It is possible to derive variants of Theorem 4.2 for $\alpha_{n}, \beta_{n} \rightarrow \infty$ in certain coupled ways. Clearly, these variants usually do not have a geometric meaning anymore. Nevertheless we discuss the case $\beta_{n} \rightarrow \infty$ and $\alpha_{n}=\beta_{n}+c$ for some constant $c \geq 0$. For $c=0$, this can be transformed by some quadratic transformation (see p.44 of [15]) into the case $\beta=-1 / 2$ and $\alpha_{n} \rightarrow \infty$ considered above. It is therefore not surprising that this transformation also appears in the following limit theorem:

4.9 Theorem. Let $c \geq 0$ be a constant, and let $\left(\beta_{n}\right)_{n \in \mathbb{N}} \subset[-1 / 2, \infty[$ be an increasing sequence of indices with $\lim _{n \rightarrow \infty} n / \sqrt{\beta}_{n}=0$. Let $\nu \in M^{1}([0, \infty[)$ with a finite second moment $\int_{0}^{\infty} x^{2} d \nu(x)<\infty$. Then, $\rho:=\int_{0}^{\infty} \ln (\operatorname{ch}(2 x)) d \nu(x)<\infty$ and $\sigma^{2}:=\int_{0}^{\infty}(\ln (\operatorname{ch}(2 x)))^{2} d \nu(x)<$ $\infty$ exist, and for the random walks $\left(S_{n}^{\left(\beta_{n}-c, \beta_{n}\right)}\right)_{n \geq 0}$ on $[0, \infty[$,

$$
\frac{2 \cdot S_{n}^{\left(\beta_{n}+c, \beta_{n}\right)}-n \rho}{\sqrt{n}}
$$

tends in distribution for $n \rightarrow \infty$ to $N\left(0, \sigma^{2}-\rho^{2}\right)$.

The proof of this second CLT is based on the observation that for $\beta \rightarrow \infty, \alpha=\beta+c$, and $f \in C([0, \infty[)$,

$$
\delta_{r} *(\alpha, \beta) \delta_{s}(f) \quad \rightarrow \quad f\left(\frac{1}{2} \operatorname{arch}(\operatorname{ch}(2 s) \operatorname{ch}(2 r))\right) ;
$$

see Lemma 4.12 below. Again, this motivates the following notions.

4.10 Definition. (1) We equip the set $[0, \infty[$ with the commutative semigroup operation

$$
s \bullet r:=\frac{1}{2} \cdot \operatorname{arch}(\operatorname{ch}(2 r) \cdot \operatorname{ch}(2 s)) \quad(r, s \geq 0),
$$

and extend this operation again in a linear, weakly continuous way to the space of all bounded Borel measures. We also notice that the mapping $Q(x):=\ln (\operatorname{ch}(2 x))$ establishes a semigroup isomorphism from $([0, \infty[, \bullet)$ onto $([0, \infty[,+)$. 
(2) A function $f \in C([0, \infty[)$ is called arche-Lipschitz continuous with constant $L$, if for all $x, y \geq 0$,

$$
\left|f\left(\frac{1}{2} \operatorname{arch}\left(e^{x}\right)\right)-f\left(\frac{1}{2} \operatorname{arch}\left(e^{y}\right)\right)\right| \leq L|x-y| .
$$

This notion is again motivated by the fact, that for a function $f \in C_{0}([0, \infty[)$, which is Lipschitz in the usual sense with constant $L$, the function $\tilde{f}(x):=f((Q(x))$ is archeLipschitz with the same $L$.

Before starting with the central parts of the proof, we note the collect some elementary equations for hyperbolic functions:

$$
\begin{gathered}
\operatorname{arch}(\sqrt{x+1 / 2})=\frac{1}{2} \cdot \operatorname{arch}(2 x) \quad(x \geq 0) \\
|\operatorname{ch} s \cdot \operatorname{ch} r+i \cdot \operatorname{sh} s \cdot \operatorname{sh} r|=\left(\frac{\operatorname{ch}(2 s) \operatorname{ch}(2 r)}{2}+1 / 2\right)^{1 / 2} \quad(s, r \geq 0) \\
\operatorname{arch}|\operatorname{ch} r \cdot \operatorname{ch} s+i \cdot \operatorname{sh} r \cdot \operatorname{sh} s|=\frac{1}{2} \operatorname{arch}(\operatorname{ch}(2 s) \operatorname{ch}(2 r)) \quad(s, r \geq 0) .
\end{gathered}
$$

The following results are the key for the proof of Theorem 4.9

4.11 Lemma. Let $f \in C([0, \infty[)$ be arche-Lipschitz continuous with constant $L$. Then, for all $r, s \geq 0, t \in[0,1]$ and $\varphi \in[0, \pi]$,

$$
\begin{aligned}
& \left|f\left(\operatorname{arch}\left|\operatorname{ch} r \cdot \operatorname{ch} s+t e^{i \varphi} \operatorname{sh} r \cdot \operatorname{sh} s\right|\right)-f(\operatorname{arch}|\operatorname{ch} r \cdot \operatorname{ch} s+i \cdot \operatorname{sh} r \cdot \operatorname{sh} s|)\right| \\
& \quad \leq 8 L \frac{\left|i-t e^{i \varphi}\right|}{\sin ^{2} \varphi} .
\end{aligned}
$$

Proof. Put

$$
A:=|\operatorname{ch} r \cdot \operatorname{ch} s+i \cdot \operatorname{sh} r \cdot \operatorname{sh} s| \quad \text { and } \quad B:=\left|\operatorname{ch} r \cdot \operatorname{ch} s+t e^{i \varphi} \operatorname{sh} r \cdot \operatorname{sh} s\right| .
$$

Using Eq. (4.12), Definition 4.10(2), and the mean value theorem for $f(x):=\ln \left(2 x^{2}-1\right)$, we obtain

$$
\begin{aligned}
\mid f(\operatorname{arch} A) & -f(\operatorname{arch} B)|=| f\left(\frac{1}{2} \operatorname{arch}\left(2 A^{2}-1\right)\right)-f\left(\frac{1}{2} \operatorname{arch}\left(2 B^{2}-1\right)\right) \mid \\
& \leq L \cdot\left|\ln \left(2 A^{2}-1\right)-\ln \left(2 B^{2}-1\right)\right| \\
& \leq 4 L \cdot|A-B| \cdot \frac{y}{2 y^{2}-1} \leq 2 L \cdot|A-B| \frac{1}{y-1 / \sqrt{2}}
\end{aligned}
$$

for some $y$ between $A$ and $B$. On the other hand,

$$
\begin{aligned}
B & \geq \operatorname{ch} r \cdot \operatorname{ch} s+t \cdot \cos \varphi \cdot \operatorname{sh} r \cdot \operatorname{sh} s \\
& =(1+(1+t \cdot \cos \varphi) \cdot \operatorname{sh} r \cdot \operatorname{sh} s=: D
\end{aligned}
$$

and $A \geq D$, and thus $y \geq D$. Moreover, we have $|A-B| \leq\left|i-t e^{i \varphi}\right| \operatorname{sh} r \cdot \operatorname{sh} s$ and

$$
1+t \cdot \cos \varphi \geq \frac{1}{2}\left(1+\cos \varphi \geq \frac{1}{4}\left(1-\cos ^{2} \varphi\right)=\frac{1}{4} \sin ^{2} \varphi .\right.
$$


We thus conclude that

$$
\begin{aligned}
|f(\operatorname{arch} A)-f(\operatorname{arch} B)| & \leq 2 L\left|i-t e^{i \varphi}\right| \frac{\operatorname{sh} r \cdot \operatorname{sh} s}{(1-1 / \sqrt{2})+(1+t \cos \varphi) \operatorname{sh} r \cdot \operatorname{sh} s} \\
& \leq 2 L \frac{\left|i-t e^{i \varphi}\right|}{1+t \cos \varphi} \leq 8 L \frac{\left|i-t e^{i \varphi}\right|}{\sin ^{2} \varphi} .
\end{aligned}
$$

4.12 Lemma. There is a constant $C=C(c)$ such that for all $r, s \in[0, \infty[$, all sufficiently large $\beta$, and all arche-Lipschitz continuous functions $f$ on $[0, \infty[$ with constant $L$,

$$
\left|\delta_{r} *_{(\beta+c, \beta)} \delta_{s}(f)-\delta_{r} \bullet \delta_{s}(f)\right| \leq C L / \sqrt{\beta} .
$$

Proof. We only consider the case $c>0$. By the definition of the Jacobi convolution, Eq. (4.14), and Lemma 4.11,

$$
\begin{aligned}
& \left|\delta_{r} *(\beta,+c, \beta) \delta_{s}(f)-\delta_{r} \bullet \delta_{s}(f)\right| \\
& \leq c_{(\beta+c, \beta)} \int_{0}^{1} \int_{0}^{\pi} \mid f\left(\operatorname{arch}\left|\operatorname{ch} r \cdot \operatorname{ch} s+t e^{i \varphi} \operatorname{sh} r \cdot \operatorname{sh} s\right|\right)- \\
& -f(\operatorname{arch}|\operatorname{ch} r \cdot \operatorname{ch} s+i \cdot \operatorname{sh} r \cdot \operatorname{sh} s|) \mid \cdot\left(1-t^{2}\right)^{c-1}(t \sin \varphi)^{2 \beta} t d t d \varphi \\
& \leq 8 L c_{(\beta+c, \beta)} \int_{0}^{1} \int_{0}^{\pi}\left|i-t e^{i \varphi}\right|\left(1-t^{2}\right)^{c-1} t^{2 \beta+1} \sin ^{2 \beta-2} \varphi d t d \varphi \\
& \leq 8 L c_{(\beta+c, \beta)} \int_{0}^{1} \int_{0}^{\pi}(\cos \varphi+(1-t \sin \varphi))\left(1-t^{2}\right)^{c-1} t^{2 \beta+1} \sin ^{2 \beta-2} \varphi d t d \varphi \\
& =8 L c_{(\beta+c, \beta)} \int_{0}^{1} \int_{0}^{\pi}(\cos \varphi+(1-t)+t(1-\sin \varphi))\left(1-t^{2}\right)^{c-1} t^{2 \beta+1} \sin ^{2 \beta-2} \varphi d t d \varphi \\
& \leq 8 L c_{(\beta+c, \beta)} \int_{0}^{1} \int_{0}^{\pi}\left(\cos \varphi+\left(1-t^{2}\right)+t \cos ^{2} \varphi\right)\left(1-t^{2}\right)^{c-1} t^{2 \beta+1} \sin ^{2 \beta-2} \varphi d t d \varphi .
\end{aligned}
$$

Using the definition of $c_{(\beta+c, \beta)}$, the beta integrals

$$
\int_{0}^{1}\left(1-t^{2}\right)^{a-1} t^{b-1} d t=\frac{\Gamma(a) \Gamma(b / 2)}{2 \Gamma(a+b / 2)}
$$

and

$$
\int_{0}^{\pi}|\cos \varphi|^{2 a-1} \sin ^{2 b-1} d \varphi=\frac{\Gamma(a) \Gamma(b)}{2 \Gamma(a+b)},
$$

and $\Gamma(x+1 / 2) / \Gamma(x)=O(\sqrt{(} x)$ for $x \rightarrow \infty$, the lemma follows readily.

The proofs of Lemmas 4.6 and 4.7, Proposition 4.8, and finally of the central limit theorem 4.2 can now be transferred word by word to this setting (by using Definition 4.10 instead of 4.3) which then leads to the proof of Theorem 4.9. 


\section{References}

[1] V. Bentkus, Dependence of the Berry-Esseen estimate on the dimension. Lithuanian Math. J. 26 (1986), 110-113.

[2] W.R. Bloom, H. Heyer, Harmonic analysis of probability measures on hypergroups. De Gruyter Studies in Mathematics 20, de Gruyter-Verlag Berlin, New York 1995.

[3] P. Diaconis, The cutoff phenomenon in finite Markov chains. Proc. Natl. Acad. Sci. USA 93, 1659-1664 (1996).

[4] P. Diaconis, R.L. Graham,J.A. Morrison,, Asymptotic analysis of a random walk on a hypercube with many dimensions. Random Struct. Algorithms 1, 51-72 (1990).

[5] J. Faraut, Analyse harmonique sur les pairs de Gelfand et les espaces hyperboliques. In: J.-L. Clerc et al.. Analyse Harmonique, C.I.M.P.A., Nice 1982, Ch. IV.

[6] J. Faraut, A. Korányi, Analysis on symmetric cones. Oxford Science Publications, Clarendon press, Oxford 1994.

[7] S. Helgason, Groups and Geometric Analysis, Academic Press 1984.

[8] C.S. Herz, Bessel functions of matrix argument. Ann. Math. 61 (1955), 474-523.

[9] A. Hora, The cut-off phenomenon for random walks on Hamming graphs with variable growth conditions. Publ. Res. Inst. Math. Sci. 33, 695-710 (1997).

[10] A. Hora, Scaling limit for Gibbs states for Johnson graphs and resulting Meixner classes. Infin. Dimens. Anal. Quantum Probab. Relat. Top. 6, 139-143 (2003).

[11] A. Hora, N.Obata, Quantum probability and spectral analysis of graphs. Springer (2007).

[12] A.T. James, Special functions of matrix and single argument in statistics. In: Theory and application of special functions. Proc. University of Wisconsin, Madison, Wis.,1975. Ed. Richard Askey. Academic Press, New York-London, 1975.

[13] R.I. Jewett, Spaces with an abstract convolution of measures, Adv. Math. 18 (1975), $1-101$.

[14] J.F.C. Kingman, Random walks with spherical symmetry. Acta Math. 109 (1963), 11-53.

[15] T. Koornwinder,Jacobi functions and analysis on noncompact semisimple Lie groups. In: Special Functions: Group Theoretical Aspects and Applications, Eds. Richard Askey et al., D. Reidel, Dordrecht-Boston-Lancaster, 1984.

[16] M. Rösler, Bessel convolutions on matrix cones, Compos. Math. 143 (2007), 749-779.

[17] M. Rösler, M. Voit, Limit theorems for radial random walks on $p \times q$ matrices as $p$ tends to infinity. To appear in Math. Nachr.. 
[18] C.Tracy, H. Widom, Distribution functions for largest eigenvalues and their applications. In: Proc. ICM 2002, Beijing, China, Ed. Ta Tsien Li, Higher Education Press, Beijing, 587-596 (2002).

[19] M. Voit, A limit theorem for isotropic random walks on $\mathbb{R}^{d}$ for $d \rightarrow \infty$. Russian J. Math. Phys. 3 (1995), 535-539.

[20] M. Voit, Asymptotic behavior of heat kernels on spheres of large dimensions. J. Multivariate Anal. 59, 230-248 (1996).

[21] M. Voit, Asymptotic distributions for the Ehrenfest urn and related random walks. $J$. Appl. Probab. 33, 340-356 (1996).

[22] M. Voit, Limit theorems for compact two-point homogeneous spaces of large dimensions. J. Theor. Probab. 9, 353-370 (1996).

[23] M. Voit, Asymptotic of heat kernels on projective spaces of large dimensions and on disk hypergroups. Math. Nachr. 194, 225-238 (1998).

[24] M. Voit, Bessel convolutions on matrix cones: Algebraic properties and random walks. arXiv: math.CA/0603017, to appear in J.Theoretical Probab..

[25] M. Voit, Central limit theorems for radial random walks on $p \times q$ matrices for $p \rightarrow \infty$. Preprint, 2008. 


\section{Preprints ab 2008}

2008-01 Henryk Zähle

Weak approximation of SDEs by discrete-time processes

2008-02 Benjamin Fine, Gerhard Rosenberger

An Epic Drama: The Development of the Prime Number Theorem

2008-03 Benjamin Fine, Miriam Hahn, Alexander Hulpke, Volkmar große Rebel, Gerhard Rosenberger, Martin Scheer

All Finite Generalized Tetrahedron Groups

2008-04

2008-05

2008-06

Ben Schweizer

Homogenization of the Prager model in one-dimensional plasticity

Benjamin Fine, Alexei Myasnikov, Gerhard Rosenberger

Generic Subgroups of Group Amalgams

Flavius Guiaş

Generalized Becker-Döring Equations Modeling the Time Evolution of a Process of Preferential Attachment with Fitness

2008-07 Karl Friedrich Siburg, Pavel A. Stoimenov

A scalar product for copulas

2008-08 Karl Friedrich Siburg, Pavel A. Stoimenov

A measure of mutual complete dependence

2008-09 Karl Friedrich Siburg, Pavel A. Stoimenov

Gluing copulas

2008-10 Peter Becker-Kern, Wilfried Hazod

Mehler hemigroups and embedding of discrete skew convolution semigroups on simply connected nilpotent Lie groups

2008-11 Karl Friedrich Siburg

Geometric proofs of the two-dimensional Borsuk-Ulam theorem

2008-12 Michael Lenzinger and Ben Schweizer

Two-phase flow equations with outflow boundary conditions in the hydrophobic-hydrophilic case

2008-13 Wilfried Hazod

Probability on Matrix-Cone Hypergroups: Limit Theorems and

Structural Properties

2008-14 Wilfried Hazod

Mixing of generating functionals and applications to (semi-)stability

of probabilities on groups 
Wilfried Hazod

Multiple selfdecomposable laws on vector spaces and on groups:

The existence of background driving processes

2008-16 Guy Bouchitté and Ben Schweizer

Homogenization of Maxwell's equations with split rings

$2008-17$

Ansgar Steland and Henryk Zähle

Sampling inspection by variables: nonparametric setting

$2008-18$

Michael Voit

Limit theorems for radial random walks on homogeneous spaces with growing dimensions 